\title{
Padrões alimentares de adolescentes na cidade de São Paulo'
}

\author{
Eating patterns of eutrophic and overweight \\ adolescents in the city of São Paulo, Brazil
}

Aline Giacomelli SALVATTI ${ }^{2}$

Maria Arlete Meil Schimith ESCRIVÃO2

José Augusto de Aguiar Carrazedo TADDEI ${ }^{2}$

Mario Maia BRACCO ${ }^{3}$

RE S U M O

\section{Objetivo}

Identificar os padrões alimentares de adolescentes eutróficos e com excesso de peso.

\section{Métodos}

Estudo transversal envolvendo 239 adolescentes de ambos os sexos, na faixa etária de 14 a 19 anos, alunos de uma escola pública da cidade de São Paulo, Brasil. Foram coletados dados antropométricos e registro alimentar de 4 dias. O estado nutricional foi avaliado pelo índice de massa corporal de acordo com os critérios de Must et al., e foi aplicada análise fatorial para a identificação dos padrões alimentares.

\section{Resultados}

Foram identificados quatro padrões alimentares: tradicional, urbano, saudável e junk food. O estado nutricional de eutrofia teve associação positiva com os padrões tradicional e urbano, o sobrepeso com os padrões saudável e junk food, e a obesidade associou-se negativamente com os padrões urbano, saudável e junk food.

\section{Conclusão}

O padrão saudável foi encontrado entre os adolescentes com sobrepeso, evidenciando preocupação com o controle do peso e tentativa de mudança do hábito alimentar. Entre os obesos, a ocorrência do sub-relato provavelmente tenha influenciado o resultado das associações.

Termos de indexação: Adolescente. Análise fatorial. Comportamento alimentar. Estado nutricional.

\footnotetext{
1 Artigo elaborado a partir da dissertação de mestrado de A. G. SALVATTI, intitulada "Comparação dos padrões de consumo alimentar entre adolescentes eutróficos e com excesso de peso". Universidade Federal de São Paulo; 2010. Apoio: o presente estudo faz parte de um projeto financiado pela Fundação de Amparo à Pesquisa do Estado de São Paulo (FAPESP), protocolo no $03 / 00415-4$.

2 Universidade Federal de São Paulo, Departamento de Pediatria. R. Loefgreen, 1647, 04040-032, São Paulo, Brasil. SP. Correspondência para/Correspondence to: J.A.A.C. TADDEI. E-mails: <taddei.dped@epm.br>; <nutsec@yahoo.com.br>.

${ }^{3}$ Centro Assistencial Cruz de Malta. São Paulo, SP, Brasil.
} 


\section{A B S T R A C T}

\section{Objective}

This study identified the dietary patterns of normal weight and overweight adolescents.

\section{Methods}

This was a cross-sectional study of 239 adolescents from a public school in São Paulo, Brazil; of both sexes, aged 14 to 19 years. Anthropometric data and 4-day dietary records were collected. Nutritional status was assessed by body mass index, according to the criteria established by Must et al. Factor analysis was used to identify dietary patterns.

\section{Results}

This assessment resulted in the identification of four dietary patterns: Traditional, Urban, Healthy, and Junk Food. Normal weight was positively associated with the Traditional and Urban patterns, and overweight was positively associated with the Healthy and Junk Food patterns. Lastly, obesity was negatively associated with the Urban, Healthy and Junk Food patterns.

\section{Conclusion}

The Healthy pattern was found among overweight adolescents who were concerned with weight control and trying to change eating habits. Among the obese, the occurrence of underreporting probably influenced the outcome of the associations.

Indexing terms: Adolescent. Factor analysis, statistical. Feeding behavior. Nutritional status.

\section{N T R O D U Ç Ã O}

Sobrepeso e obesidade na infância e na adolescência são importantes problemas de saúde pública, com aumento crescente da incidência e da prevalência em todo o mundo. Dados da Organização Mundial da Saúde (OMS) indicam que, na população mundial, 10,0\% das crianças e dos adolescentes entre cinco e 17 anos apresentam excesso de peso ${ }^{1}$. Entre os adolescentes brasileiros de 10 a 19 anos, 19,4\% das meninas e $21,7 \%$ dos meninos encontram-se nessas mesmas condições ${ }^{2}$.

A obesidade iniciada na infância e na adolescência pode permanecer na fase adulta e levar ao desenvolvimento das doenças crônicas não transmissíveis, como o diabetes tipo 2 e as doenças cardiovasculares, reduzindo a qualidade e a expectativa de vida ${ }^{1,3}$. As doenças crônicas não transmissíveis são as principais causas de morte em todo o mundo, inclusive nos países de baixa e média renda, ultrapassando em mortalidade as doenças infecciosas ${ }^{4}$.

As crianças e os adolescentes já podem apresentar as consequências da obesidade, como problemas osteoarticulares, apneia do sono, disli- pidemias, hipertensão arterial, alterações do metabolismo da glicose, além dos prejuízos psicossociais provocados pelo estigma dessa doença $a^{1,3,4}$.

O aumento da prevalência de obesidade na infância e na adolescência é influenciado por mudanças no estilo de vida e nos hábitos alimentares. Entre as mudanças ocorridas na alimentação da população brasileira, destaca-se o aumento do consumo de alimentos e bebidas industrializados, ricos em açúcar e gorduras, que apresentam, em geral, alto teor energético. Paralelamente ao aumento do consumo desses alimentos, observa-se a diminuição da ingestão de frutas, verduras e legumes 5 . O tamanho das porções dos alimentos industrializados também tem aumentado de maneira expressiva ao longo do tempo ${ }^{6}$.

Estudos realizados com a população brasileira revelam mudanças nos hábitos alimentares $^{7,8}$. Entre os adolescentes, vem ocorrendo substituição de alimentos tradicionais, como o arroz e o feijão, por alimentos de baixo conteúdo nutricional e alta concentração energética, como os fast foods e as guloseimas ${ }^{6}$.

Sanchez-Villegas et al. ${ }^{9}$, analisando padrões alimentares em população espanhola, 
verificaram que adolescentes mais velhos apresentam uma maior tendência de adotar o padrão de consumo junk food. Isso pode ser atribuído ao fato de o adolescente estar em fase de contestação e busca por independência, o que propicia o rompimento com os hábitos alimentares tradicionais da família. O maior número de compromissos e a necessidade de identificação com seus pares também costumam afetar a escolha alimentar do adolescente ${ }^{10}$.

O hábito alimentar é determinado pela acessibilidade e pela disponibilidade dos alimentos, pelo nível socioeconômico, entre outros fatores $^{10}$. Dificilmente variáveis associadas apenas ao indivíduo explicam as escolhas alimentares.

Os hábitos alimentares inadequados, adquiridos na infância e na adolescência, podem exercer grande influência no desenvolvimento das doenças crônicas não transmissíveis ${ }^{11}$, mas a relação entre dieta e doença nem sempre é detectada nos estudos epidemiológicos devido às dificuldades de se avaliar com precisão a ingestão alimentar ${ }^{6}$.

As avaliações do consumo alimentar geralmente são baseadas no cálculo da ingestão energética total, dos macro e micronutrientes, ou na análise de um único nutriente ${ }^{12}$. Porém, os indivíduos não consomem nutrientes isoladamente e sim refeições compostas por grande variedade de nutrientes, que sofrem interações - facilitando ou dificultando sua absorção - que dificultam a detecção dos efeitos de cada nutriente separadamente. As preparações e o cozimento também influenciam o valor nutricional dos alimentos. Somando-se a isso, existe a dificuldade de se mensurar a dieta habitual de forma acurada e precisa, devido às limitações dos instrumentos disponíveis e ao viés do informante ${ }^{6,13}$

O estudo de conjuntos ou grupos de alimentos tem a vantagem de superar parte dessas limitações e expressar melhor a dieta consumida por uma dada população ${ }^{6}$. A identificação desses conjuntos ou grupos de alimentos é feita por meio de métodos estatísticos de redução e/ou agregação de componentes, formando os padrões ali- mentares $^{14}$. Os padrões alimentares podem ser detectados pela análise fatorial, que utiliza as correlações entre os vários alimentos consumidos para descrever o padrão alimentar geral ${ }^{6}$. Essa análise costuma ser realizada para avaliar o efeito positivo ou negativo de múltiplos fatores alimentares na saúde dos indivíduos ${ }^{15}$.

Um dos primeiros estudos que trabalharam com padrões alimentares foi o de Schwerin et al. ${ }^{16}$, que avaliou a ingestão alimentar da população americana, usando a análise fatorial para converter o consumo alimentar em algo mais significativo do que componentes isolados da dieta. A partir daí, outros estudos associaram padrões alimentares com fatores de risco à saúde 15,17-19

A OMS recomenda o estudo de grupos de alimentos ao invés de nutrientes isoladamente ${ }^{20}$. A avaliação de padrões de consumo alimentar, mais do que a ausência ou a presença específica de nutrientes, indica a capacidade da dieta em promover saúde ou desenvolver doenças na população estudada ${ }^{15}$, podendo gerar conhecimentos para subsidiar o planejamento de medidas de promoção da saúde ${ }^{21}$. O estudo dos padrões de consumo alimentar também permite avaliar a disponibilidade de alimentos e a inserção da população nos diferentes cenários socioeconômicos ${ }^{8}$.

Considerando as modificações dos hábitos alimentares das últimas décadas, a dificuldade de se analisarem nutrientes isoladamente e a escassez de estudos na literatura relacionando padrões de consumo alimentar e estado nutricional em adolescentes, o presente estudo teve como objetivos identificar os padrões de consumo alimentar de uma população de adolescentes e verificar possíveis associações com o estado nutricional.

\section{M É T O D O S}

Este estudo faz parte do Projeto Estudos Clínicos sobre Crescimento, Comportamento, Hipertensão Arterial, Obesidade e Saúde Bucal $(\mathrm{ECCCHOS})^{13}$, que teve como objetivo detectar fatores de risco e morbidades associados ao 
excesso de peso em adolescentes matriculados em uma escola pública, na cidade de São Paulo, por meio de avaliações clínicas, antropométricas, laboratoriais, de hábitos alimentares e de estilo de vida. O delineamento do Projeto ECCCHOS foi do tipo caso-controle, em que os casos foram representados pelos adolescentes com sobrepeso ou obesidade, e os controles, pelos adolescentes eutróficos. Para cálculo da amostra, considerouse a prevalência de obesidade familiar entre os controles de $25 \%$. Assumindo poder de $80 \%$ e erro tipo 1 de 5\%, a amostra resultou em 65 casos e 65 controles, com acréscimos de $10 \%$ para as eventuais perdas e $10 \%$ para a análise estratificada, chegando-se ao total de 78 casos e 78 controles.

Foram pesados e medidos 1420 adolescentes (98,68\% do total de alunos) de uma escola pública. A seleção dos adolescentes para o estudo seguiu a ordem da lista de chamada. Adolescentes gestantes e lactantes não foram incluídas na amostra. Os eutróficos foram escolhidos a partir da seleção de obesos ou sobrepesos para o pareamento, segundo sexo, idade e série, e constituíram o grupo-controle. Foram elegíveis para o estudo 340 adolescentes; destes, 77 se recusaram a participar. Dos 263 adolescentes que aceitaram participar do projeto, 13 foram excluídos por apresentarem doenças agudas ou outras doenças crônicas além da obesidade, totalizando 250.

No presente estudo, 11 adolescentes foram excluídos por não terem preenchido o registro alimentar de quatro dias corretamente. Dessa forma, foram analisados os dados antropométricos e nutricionais de 239 adolescentes de 14 a 19 anos, sendo 128 meninas (53,56\%) e 111 meninos $(46,44 \%)$, em estágio de desenvolvimento puberal 4 ou 5 , segundo os critérios de Tanner ${ }^{22}$. A amostra foi dividida em 3 grupos de acordo com o estado nutricional: eutróficos, com sobrepeso e obesos.

Para determinação do peso corporal, foi utilizada uma balança eletrônica tipo plataforma, da marca Kratos ${ }^{\circledR}$, modelo "Linea", com capacidade mínima de $1,25 \mathrm{~kg}$ e máxima de $150 \mathrm{~kg}$, e com precisão de 50 gramas, disposta em superfície firme e plana. A balança era aferida antes de cada pesagem. Os adolescentes foram pesados descalços e trajando roupas leves. Na determinação da estatura foi utilizado um estadiômetro portátil da marca Alturexata ${ }^{\circledR}$, com escala em milímetros, fixado em superfície perpendicular ao solo. Os adolescentes ficavam descalços, com os pés unidos, joelhos esticados, cabeça orientada no plano horizontal de Frankfurt, sendo as medidas realizadas duas vezes, utilizando-se a média como valor final. Os valores do peso corporal foram expressos em quilogramas e os da altura em metros, e utilizados para calcular o Índice de Massa Corporal $(\mathrm{IMC})=$ peso, em quilos, dividido pela estatura, em metros, ao quadrado.

Para a classificação do estado nutricional dos adolescentes, foram utilizados os critérios de Must et al. ${ }^{23}$, considerando como eutrofia, os valores de IMC acima do percentil 5 e abaixo do percentil 85; como sobrepeso, os valores de IMC entre os percentis 85 e abaixo de 95, e como obesidade, os valores a partir do percentil $95^{23}$.

O consumo alimentar foi estimado por meio do registro alimentar de 4 dias, sendo 3 dias durante a semana e um no final de semana. A avaliação do consumo energético (em quilocalorias) e a distribuição dos macronutrientes (em gramas) foram obtidas utilizando-se o Nutwin (Programa de Apoio à Nutrição, versão 1.5, Universidade Federal de São Paulo, São Paulo; 2002). Foram inseridos os alimentos que não apresentavam informações nutricionais registradas no programa, utilizando-se tabelas de composição centesimal que contemplavam seus nutrientes; para os alimentos industrializados, foram utilizadas as informações nutricionais contidas em seus rótulos.

Para a análise dos padrões de consumo alimentar, os itens encontrados nos registros foram agrupados de acordo com a semelhança do conteúdo nutritivo ou composição botânica ${ }^{14}$, resultando em 30 grupos de alimentos (Tabela 1). As análises foram focadas no consumo dos alimentos relatados e na sua identificação nos grupos de alimentos. 
Tabela 1. Grupos de alimentos de acordo com o conteúdo nutritivo ou a composição botânica, em adolescentes de uma Escola Pública, na cidade de São Paulo (SP), 2010.

\begin{tabular}{|c|c|}
\hline Alimento ou grupo de alimentos & Alimentos encontrados nos registros alimentares \\
\hline 1. Arroz & Arroz branco e integral. \\
\hline 2. Feijões & Feijão, ervilha, lentilha, grão-de-bico e soja. \\
\hline 3. Farinhas & Farinha de mandioca, milho e farofas. \\
\hline 4. Tubérculos & Batata, batata-doce, mandioca, inhame, cará. \\
\hline 5. Carne vermelha & Carne bovina, suína, miúdos. \\
\hline 6. Óleos & Óleos e azeite. \\
\hline 7. Leite e derivados & $\begin{array}{l}\text { Leite integral, semi-desnatado, queijos amarelos, requeijão, iogurtes com frutas e leite } \\
\text { fermentado. }\end{array}$ \\
\hline 8. Pães & Pão francês, de forma, sovado, integral, biscoito salgado, torradas \\
\hline 9. Leite desnatado & Leite desnatado. \\
\hline 10. logurte desnatado & logurte desnatado. \\
\hline 11. Bolachas, bolos e tortas doces & Bolachas recheadas, bolos e tortas doces. \\
\hline 12. Embutidos & Salsicha e linguiça. \\
\hline 13. Manteiga e margarina & Manteiga e margarinas. \\
\hline 14. Achocolatados & Achocolatados em pó, achocolatado diet, leite achocolatado \\
\hline 15. Condimentos & Maionese, catchup, mostarda. \\
\hline 16. Refrigerantes & Refrigerante normal e diet e chás industrializados. \\
\hline 17. Macarrão instantâneo & Macarrão instantâneo. \\
\hline 18. Verduras & Alface, repolho, rúcula, agrião, escarola, almeirão, acelga, couve e espinafre. \\
\hline 19. Legumes & Tomate, cenoura, cebola, berinjela, beterraba, vagem, chuchu, pepino, abobrinha, \\
\hline 20. Frutas & $\begin{array}{l}\text { Maçã, banana, pêra, mamão, morango, uva, mexerica, laranja, melancia, kiwi, ameixa, } \\
\text { abacaxi, abacate, goiaba, figo, manga. }\end{array}$ \\
\hline 21. Suco natural & Sucos naturais, sem adição de açúcar. \\
\hline 22. Carnes brancas & Carne de aves e peixes, cozidas ou grelhadas. \\
\hline 23. Açúcar & Açúcar de adição. \\
\hline 24. Bebidas alcoólicas & Cerveja, uísque, vinho, vodca, cachaça e licor. \\
\hline 25. Sobremesa láctea & Flans e pudins industrializados. \\
\hline 26. Café e capuccino & Café e capuccino. \\
\hline 27. Doces & Bala, chiclete, gelatina, geleia, leite condensado. \\
\hline 28. Chocolate e sorvete cremoso & Chocolates e sorvetes cremosos. \\
\hline 29. Picolé & Picolés à base de frutas. \\
\hline 30. Frituras & Salgados fritos, pipoca, frango empanado industrializado, hambúrguer, batata frita, bacon. \\
\hline
\end{tabular}

Para a denominação dos padrões foi utilizada nomenclatura idêntica ou semelhante à de outros estudos realizados, com a mesma metodologia, na população brasileira17,19.

\section{Análise estatística}

Os questionários foram previamente avaliados quanto à sua consistência, e as questões abertas foram codificadas e posteriormente os dados foram digitados no software "Epi Info" (Epi Info, versão 6.0, Centers for Disease Control and Prevention, Atlanta, GA, USA;1994). Os dados foram duplamente digitados e validados com vistas à correção de eventuais erros.

Os padrões alimentares foram identificados pela Análise Fatorial por Componentes Principais (ACP), utilizando-se o programa Statistical 
Package for the Social Science (SPSS, versão 13.0, Chicago, IL, USA; 1999). Essa técnica foi utilizada com o objetivo de identificar padrões alimentares a partir de medidas-resumo (grupos de alimentos apresentados na Tabela 1), considerando-se a média da quantidade consumida nos 4 dias de cada alimento/preparação. A ingestão alimentar foi ajustada pelo consumo total de energia, usando-se o método residual ${ }^{24}$.

Esta análise foi dividida em etapas: preparação da matriz de correlação, extração de um conjunto de fatores da matriz de correlação, deter- minação do número de fatores e a rotação dos fatores para aumentar sua interpretabilidade ${ }^{14}$. As combinações de variáveis observadas em cada fator, em especial os itens que apresentavam carga fatorial maior, foram importantes para a interpretação e denominação dos fatores.

O primeiro passo da análise foi verificar se as variáveis eram métricas e se o tamanho da amostra era igual ou maior do que $150^{12,14}$. Para cada variável estudada, deve-se ter, no mínimo, 5 indivíduos ${ }^{14}$. No presente estudo, o número total da amostra foi de 239 indivíduos, com 30 variáveis

Tabela 2. Distribuição da carga fatorial dos alimentos nos quatro padrões de consumo alimentar, em adolescentes de uma Escola Pública, na cidade de São Paulo (SP), 2010.

\begin{tabular}{|c|c|c|c|c|}
\hline \multirow{2}{*}{ Alimento ou grupo de alimentos } & \multicolumn{4}{|c|}{ Padrões } \\
\hline & Tradicional & Urbano & Saudável & Junk food \\
\hline 1. Arroz & 0,717 & 0,058 & 0,041 & 0,168 \\
\hline 2. Feijões & 0,559 & 0,038 & 0,068 & 0,153 \\
\hline 3. Farinhas & 0,258 & 0,051 & 0,002 & 0,115 \\
\hline 4. Tubérculos & 0,326 & 0,399 & 0,117 & 0,048 \\
\hline 5. Carne vermelha & 0,464 & 0,019 & 0,230 & 0,125 \\
\hline 6. Óleos & 0,735 & 0,040 & 0,239 & 0,072 \\
\hline 7. Leite integral e derivados & 0,260 & 0,401 & 0,190 & 0,351 \\
\hline 8. Pães & 0,099 & 0,572 & 0,084 & 0,184 \\
\hline 9. Leite desnatado & 0,149 & 0,104 & 0,607 & 0,204 \\
\hline 10. logurte desnatado & 0,116 & 0,039 & 0,515 & 0,191 \\
\hline 11. Bolachas, bolos e tortas doces & 0,032 & 0,281 & 0,123 & 0,013 \\
\hline 12. Embutidos & 0,152 & 0,642 & 0,047 & 0,77 \\
\hline 13. Manteiga e margarina & 0,121 & 0,501 & 0,082 & 0,244 \\
\hline 14. Achocolatados & 0,149 & 0,464 & 0,150 & 0,165 \\
\hline 15. Condimentos & 0,140 & 0,498 & 0,195 & 0,338 \\
\hline 16. Refrigerantes & 0,136 & 0,244 & 0,093 & 0,362 \\
\hline 17. Macarrão instantâneo & 0,131 & 0,294 & 0,049 & 0,015 \\
\hline 18. Verduras & 0,232 & 0,133 & 0,468 & 0,057 \\
\hline 19. Legumes & 0,133 & 0,187 & 0,422 & 0,201 \\
\hline 20. Frutas & 0,232 & 0,000 & 0,425 & 0,171 \\
\hline 21. Suco natural & 0,086 & 0,124 & 0,254 & 0,105 \\
\hline 22. Carnes brancas & 0,015 & 0,010 & 0,581 & 0,094 \\
\hline 23. Açúcar & 0,031 & 0,108 & 0,012 & 0,408 \\
\hline 24. Bebidas alcoólicas & 0,022 & 0,097 & 0,105 & 0,298 \\
\hline 25. Sobremesa láctea & 0,220 & 0,028 & 0,131 & 0,310 \\
\hline 26. Café e capuccino & 0,006 & 0,047 & 0,107 & 0,342 \\
\hline 27. Doces & 0,207 & 0,076 & 0,091 & 0,360 \\
\hline 28. Chocolate e sorvete & 0,195 & 0,037 & 0,065 & 0,372 \\
\hline 29. Picolé & 0,105 & 0,058 & 0,109 & 0,342 \\
\hline 30. Frituras & 0,039 & 0,058 & 0,125 & 0,496 \\
\hline
\end{tabular}

* Método de Extração: Análise por componentes principais.

* Método de Rotação: Varimax com normalização Kaiser.

Nota: os valores em negrito indicam o padrão alimentar que encontra predomínio de cada grupo de alimentos. 
(grupos de alimentos); portanto, a razão encontrada entre o número de casos e o número da amostra foi igual a 7,96, excedendo o número mínimo requerido.

A matriz de correlação de variáveis deve conter duas ou mais correlações iguais ou maiores do que 0,3. Nesse estudo, 25 variáveis apresentaram correlação acima desse valor.

A adequação dos dados para a análise fatorial foi confirmada pelos testes Kaiser-Meyer-Olkin Measure of Sampling Adequacy (KMO) e Bartlett Test of Sphericity (BTS) ${ }^{12,14}$. O valor encontrado para o KMO foi de 0,703, indicando bom ajuste da análise fatorial; a significância para o BTS foi menor que 0,001 , permitindo novamente confirmar a possibilidade e a adequação do método de análise fatorial para o tratamento dos dados.

Também foi verificada a adequação de cada variável à análise fatorial, utilizando-se a matriz de correlação anti-imagem, em cuja diagonal as medidas de adequação das variáveis são alocadas. A medida de adequação deve ser maior do que 0,50 para cada variável do estudo.

Após essas verificações, foi selecionada a rotação Varimax para a obtenção de fatores não correlacionados entre si. O número de fatores a serem extraídos na análise foi definido pelo método de análise paralela, com o objetivo de se obter um número de fatores que possibilitasse a identificação de padrões interpretáveis ${ }^{25}$. Utilizando-se a matriz fatorial rotacionada, foram gerados 4 fatores (padrões alimentares), represen- tando o consumo alimentar da população em estudo (Tabela 2).

A última etapa consistiu no cálculo dos escores fatoriais para cada indivíduo, possibilitando que cada um tivesse um escore fatorial em todos os padrões identificados e posicionando-o segundo o grau de participação em cada padrão ${ }^{12,14}$. As associações entre os escores fatoriais dos diferentes padrões alimentares e as três condições nutricionais (eutrofia, sobrepeso e obesidade) foram avaliadas pela análise de variância, sem estratificação por sexo, adotando-se o nível de significância de 5\%.

O estudo foi aprovado pelo Comitê de Ética em Pesquisa da Universidade Federal de São Paulo - processo número 0028/10, e todos os participantes assinaram um termo de consentimento livre e esclarecido antes de sua inclusão na amostra.

\section{RES U L T A D O S}

\section{Características gerais da amostra}

A Tabela 3 descreve características da amostra - idade, dados antropométricos e consumo alimentar - de acordo com a condição nutricional. O consumo energético foi semelhante nos três grupos (eutróficos, sobrepesos e obesos). O consumo de carboidratos e lipídeos foi significantemente menor nos dois grupos com excesso de peso e o de proteínas foi maior no grupo de obesos (Tabela 3).

Tabela 3. Ingestão energética e de macronutrientes, de acordo com a condição nutricional, em adolescentes de uma Escola Pública, na cidade de São Paulo (SP), 2010.

\begin{tabular}{|c|c|c|c|c|c|c|c|}
\hline & \multicolumn{2}{|c|}{ Eutróficos $(n=157)$} & \multicolumn{2}{|c|}{ Sobrepesos $(n=38)$} & \multicolumn{2}{|c|}{ Obesos $(n=44)$} & \multirow{2}{*}{$p$} \\
\hline & $M$ & DP & $M$ & DP & $M$ & DP & \\
\hline Idade (anos) & 16,25 & 0,97 & 16,11 & 0,89 & 15,93 & 1,04 & 0,234 \\
\hline IMC $\left(\mathrm{kg} / \mathrm{m}^{2}\right)$ & 20,45 & 2,10 & 27,33 & 1,26 & 32,01 & 2,56 & $0,000^{*}$ \\
\hline VET (kcal) & 2086,51 & 601,94 & 1878,00 & 789,44 & 1966,35 & 547,65 & 0,534 \\
\hline Carboidrato (g) & 281,68 & 117,31 & 240,86 & 99,7 & 252,73 & 88,12 & $0,000^{*}$ \\
\hline Proteína (g) & 75,91 & 27,98 & 74,43 & 39,48 & 78,56 & 22,01 & $0,000^{*}$ \\
\hline Lipídeos (g) & 73,74 & 39,50 & 59,31 & 37,35 & 64,64 & 27,18 & $0,000^{*}$ \\
\hline
\end{tabular}

*Valor de p<0,05; VET: valor energético total diário; M: média; DP: desvio-padrão. 


\section{Padrões alimentares da amostra estudada}

Foram identificados quatro padrões alimentares, representando o consumo da população em estudo, explicando 33,56\% da variabilidade total dos dados.

As características de cada fator, como a denominação, a variância total correspondente e os alimentos que contribuíram de forma mais relevante, estão descritas abaixo:

Padrão 1 (tradicional): explicou 10,95\% da variância total. Nesse fator, os alimentos que contribuíram de forma significativa foram: arroz, feijão e outras leguminosas, farinhas, carne bovina e óleos.

Padrão 2 (urbano): correspondeu a 9,11\% da variância total. Os alimentos incorporados a esse fator foram: tubérculos, leite (integral e semidesnatado) e derivados, pães, bolachas, bolos e tortas doces, embutidos, manteiga e margarina, achocolatados, condimentos e macarrão instantâneo.

Padrão 3 (saudável): representou 8,02\% da variância total. Os alimentos que mais contribuíram para esse fator foram: leite e iogurte desnatados, verduras, legumes, frutas, sucos naturais, carnes de aves e peixes.

Padrão 4 (junk food): representou 5,51\% da variância total. Os alimentos que compõem esse fator são: refrigerantes, açúcar, bebida alcoólica, sobremesa láctea, cafés, doces, sorvete, chocolate, picolé de frutas e frituras.

\section{Padrões alimentares e estado nutricional}

Verificou-se que os adolescentes com estado nutricional de eutrofia apresentavam associação significante e positiva com os padrões tradicional e urbano, e negativa com os padrões saudável e junk food. Os adolescentes com sobrepeso tiveram associação significante e positiva com os padrões saudável e junk food; os obesos, associação positiva apenas com o padrão tradicional e inversa com os outros padrões (Figura 1).

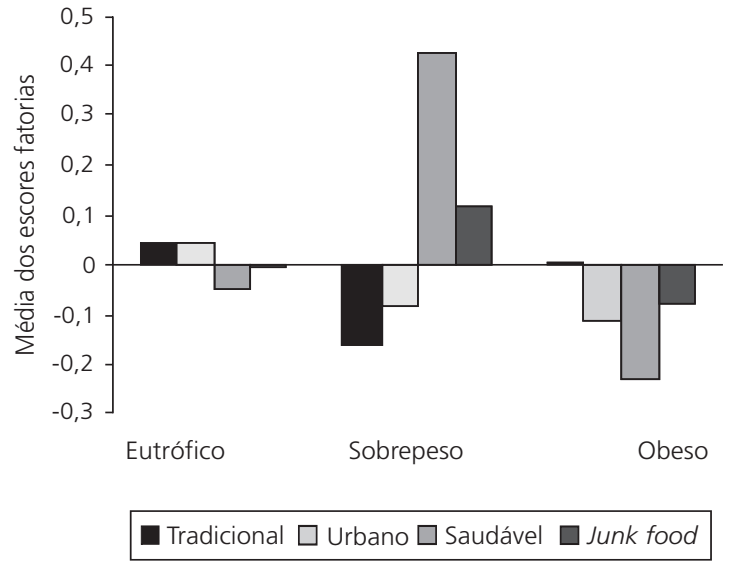

Figura 1. Distribuição das médias dos escores fatoriais para os quatro padrões alimentares, segundo os grupos de condição nutricional, em adolescentes de uma escola pública, na cidade de São Paulo (SP), 2010

\section{I S C U S S Ã O}

O presente estudo permitiu caracterizar quatro padrões alimentares, que representam o consumo da população em análise e explicam $33,56 \%$ da variabilidade total dos dados, o que corrobora outros estudos encontrados na literatura com o mesmo número de padrões ${ }^{15,26}$.

O padrão tradicional, caracterizado por alimentos presentes na alimentação dos brasileiros, como arroz, feijão, carne e farinha, apresenta semelhanças com o padrão de nomenclatura idêntica de outros estudos ${ }^{8,15,17}$. O grupo que apresentou maior aderência a esse padrão foi o de eutróficos, e a menor aderência foi verificada entre os adolescentes com sobrepeso. Estudos semelhantes realizados com adultos revelaram que a dieta tradicional brasileira, baseada em arroz e feijão, protege contra o excesso de peso ${ }^{8,19,27}$.

O padrão urbano foi constituído por leite e derivados, pães, embutidos, bolachas, achocolatados, macarrão instantâneo, que são alimentos de fácil preparo e bastante consumidos pelos adolescentes em substituição aos alimentos presentes no padrão tradicional. Esse padrão também obteve média de escores fatoriais individuais maiores 
no grupo de adolescentes eutróficos. Nos grupos com sobrepeso e obesidade, o padrão urbano apresentou baixa aderência, talvez pela omissão do consumo dos alimentos desse padrão pelos adolescentes com excesso de peso. Resultado diferente foi encontrado por Shin et al. ${ }^{28}$, que verificaram que o alto consumo de macarrão instantâneo e embutidos era determinante para o excesso de peso em crianças coreanas.

O padrão alimentar saudável recebeu essa denominação por ser composto por alimentos ricos em vitaminas, minerais, fibras, gorduras mono e poli-insaturadas e com baixos teores de açúcares, gorduras trans e saturadas. O padrão saudável foi mais consumido pelo grupo que apresentava sobrepeso. Uma hipótese para explicar esse achado seria a tentativa de controle do peso corporal pela mudança do hábito alimentar com o consumo de alimentos saudáveis.

Okubo et al. ${ }^{29}$, estudando padrões alimentares de mulheres japonesas de 18 a 20 anos, encontraram correlações positivas entre padrão saudável e maiores consumos de vitamina $C$, potássio, magnésio, cálcio e fibra dietética, e correlações negativas com carga e índice glicêmicos.

O padrão denominado junk food foi caracterizado pela alta ingestão de guloseimas como chocolates, sorvetes, açúcar de adição, frituras (batata frita, hambúrguer, empanados, pipoca, bacon), refrigerantes e bebidas alcoólicas. Esse padrão foi menos consumido pelos adolescentes eutróficos e mais por aqueles que apresentavam sobrepeso. Os alimentos desse padrão têm alta densidade energética, são ricos em açúcares, gorduras saturadas e trans e sódio e são considerados de risco para o desenvolvimento das doenças crônicas não transmissíveis, como o diabetes tipo 2 e as doenças cardiovasculares ${ }^{17}$.

Um estudo semelhante realizado com crianças e adolescentes coreanos encontrou associação entre o padrão "pizza e drinks" e maior percentual de gordura corporal entre as meninas $^{26}$. O padrão junk food pode estar relacionado à alimentação fora de casa e às outras características da escolha alimentar do adolescente, como boa palatabilidade, aceitação, status e praticidade na preparação, além da influência da mídia, que anuncia mais as guloseimas e os alimentos e bebidas presentes nos restaurantes do tipo fast-food ${ }^{17,30}$

O grupo de indivíduos obesos apresentou pouca aderência a todos os padrões identificados. Foi observada associação positiva apenas com o padrão tradicional, embora com menor média de escore fatorial em comparação com o grupo de eutróficos. Isso pode ser explicado pelo sub-relato, que é o não relato de alimentos de fato consumidos no registro alimentar, ou pela redução e/ou modificação do consumo habitual nos dias da coleta de dados, comportamentos frequentemente observados entre obesos.

A relação entre consumo alimentar e obesidade nem sempre é evidenciada em estudos transversais. Alguns autores verificaram que indivíduos obesos consumiam quantidades iguais ou menores do que as consumidas pelos eutróficos, revelando tendência dos obesos ao sub-relato ${ }^{8,31}$. No presente estudo, também não foram observadas diferenças no consumo energético entre os três estados nutricionais, e o consumo de carboidratos e lipídeos foi menor nos grupos com excesso de peso. Summerbell et al..$^{32}$ analisaram a relação entre estado nutricional e padrões de consumo alimentar, observando subestimação do consumo de lanches e da ingestão calórica total no grupo de adolescentes obesos, principalmente entre as meninas. Da mesma forma, Craig et al. ${ }^{33}$ observaram, em crianças escocesas, menor consumo dos alimentos do padrão snacks, que equivale ao padrão junk food encontrado neste estudo entre os meninos obesos.

Uma limitação do estudo, comum a todos que avaliam consumo alimentar, foi a utilização de inquéritos dietéticos, considerados de baixa acurácia. Entretanto, tais instrumentos são habitualmente utilizados em estudos populacionais. Uma alternativa seria o uso de marcadores bioquímicos, porém as técnicas são extremamente dispendiosas e avaliam um nutriente de cada vez ${ }^{34}$. Considerando que não existe um método ideal 
para avaliação do consumo alimentar, neste tipo de estudo, o registro alimentar foi escolhido por não recorrer à memória do indivíduo e ser um formulário aberto, que acomoda número ilimitado de alimentos ${ }^{12}$. O registro alimentar pode ser feito em período de 1 a 7 dias, mas recomenda-se não ultrapassar 3 a 4 dias para evitar a fadiga do entrevistado ${ }^{35}$. Além disso, há evidências de que exista menor sub-relato no registro alimentar quando comparado ao questionário de frequência alimentar, que avalia o consumo retrospectivo ${ }^{36}$.

\section{O N CLUS Ã O}

Entre os adolescentes eutróficos foi encontrado maior consumo de alimentos tradicionais da alimentação brasileira e menor ingestão de guloseimas e bebidas industrializadas, evidenciando que esse hábito alimentar pode ser protetor contra o excesso de peso.

Nos indivíduos com sobrepeso, apesar do consumo de alimentos do padrão junk food, houve predomínio de ingestão dos alimentos do padrão saudável, sugerindo preocupação com o controle de peso e tentativa de mudança do hábito alimentar nesse grupo.

Nos adolescentes obesos, a presença do sub-relato provavelmente tenha influenciado as associações entre os padrões alimentares e o estado nutricional.

\section{COLABORADORES}

A.G. SALVATTI participou da concepção do trabalho, da coleta, da análise e da interpretação dos resultados e da redação do artigo. M.A.M.S. ESCRIVÃO participou da concepção do trabalho, da interpretação dos resultados e da redação do artigo. J.A.A.C. TADDEI participou da concepção do trabalho, interpretação dos resultados e revisão crítica do artigo. M.M. BRACCO participou da revisão crítica do artigo.

\section{REFERÊ NCIAS}

1. World Health Organization. Fight childhood obesity to help prevent diabetes, say WHO \& IDF.2004 [cited 2010 Mar 30]. Available from: <http://www. who.int/mediacentre/news/releases/2004/pr81/en/ index.html>.

2. Instituto Brasileiro de Geografia e Estatística. Pesquisa de Orçamentos Familiares 2008-2009: antropometria e estado nutricional de crianças, adolescentes e adultos no Brasil. Rio de Janeiro: IBGE; 2010.

3. Cimadon HMS, Geremia R, Pellanda LC. Dietary habits and risk factors for atherosclerosis in students from Bento Gonçalves (state of Rio Grande do Sul). Arq Bras Cardiol. 2010; 95(2):166-72. doi: 10.1 590/50066-782X2010005000088.

4. Organização Mundial de Saúde. Prevenção de doenças crônicas: um investimento vital. 2005 [acesso 2010 mar 29]. Disponível em: <http://www. who.int/chp/chronic_disease_report/part1_port. pdf $>$.

5. Toral N, Slater B, Silva MV. Consumo alimentar e excesso de peso de adolescentes de Piracicaba, São Paulo. Rev Nutr. 2007; 20(5):449-59. doi: 10.1590/ S1415-52732007000500001.

6. Kac G, Sichieri R, Gigante DP, organizadores. Epidemiologia nutricional. Rio de Janeiro: Fiocruz; 2007. p.347-57.

7. Monteiro CA, Mondini L, Costa RBL. Mudanças na composição e adequação nutricional da dieta familiar nas áreas metropolitanas do Brasil. Rev Saúde Pública. 2000; 34(3):251-8.

8. Sichieri R, Castro JFG, Moura AS. Fatores associados ao padrão de consumo alimentar da população brasileira urbana. Cad Saúde Pública. 2003; 19(Supl 1):S47-53. doi: 10.1590/S0102-311X200300070 0006.

9. Sanchez-Villegas A, Delgado-Rodriguez $M$, Martinez-Gonzalez MA, De Irala-Estevez J. Gender, age, socio demographic and lifestyle factors associated with major dietary patterns in the Spanish Project SUN (Seguimiento Universidad de Navarra). Eur J Clin Nutr. 2003; 57(2):285-92. doi: 10.1038/sj.ejcn. 1601528

10. Story M, Neumark-Sztainer D, French S. Individual and environmental influences on adolescent eating behaviors. J Am Diet Assoc. 2002; 102(3 Suppl): S40-S51.

11. Willet WC. Overview and perspective in human nutrition. Asia Pac J Clin Nutr. 2008; 17(S1):1-4.

12. Fisberg RM, Slater $B$, Martini LA. Métodos de inquéritos alimentares. In: Fisberg RM, Slater $B$, Marchioni DML, Martini LA. Inquéritos alimentares: métodos e bases científicos. Barueri: Manole; 2005. p. 1-31.

13. Silveira D, Taddei JAAC, Escrivão MAMS, Oliveira FLCO, Ancona-Lopez F. Risk factors for overweight 
among Brazilian adolescents of low-income families: a case-control study. Public Health Nutr. 2006; 9(4):421-28. doi: 10.1079/PHN2005875.

14. Hu FB. Dietary pattern analysis: a new direction in nutritional epidemiology. Curr Opin Lipidol. 2002; 13(1):3-9.

15. Neumann AICP, Martins IS, Marcopito LF, Araujo EAC. Padrões alimentares associados a fatores de risco para doenças cardiovasculares entre residentes de um município brasileiro. Rev Panam Salud Publica. 2007; 22(5):329-39. doi: 10.1590/S1020-4 9892007001000006.

16. Schwerin HS, Stanton JL, Smith JL, Riley AM, Brett BE. Food, eating habits, and health: a further examination of the relationship between food eating patterns and nutritional health. Am J Clin Nutr. 1982; 35(5 Suppl):1319-25.

17. Dishchekenian VRM. Padrões alimentares de adolescentes obesos e suas repercussões metabólicas [dissertação]. São Paulo: Universidade Federal de São Paulo; 2009.

18. McNaughton SA, Ball K, Mishra GD, Crawford DA. Dietary patterns of adolescents and risk of obesity and hypertension. J Nutr. 2008; 138(2):364-70.

19. Sichieri R. Dietary patterns and their associations with obesity in the Brazilian city of Rio de Janeiro. Obes Res. 2002; 10(1):42-49. doi: 10.1590/S0102$311 \times 2010000100014$.

20. World Health Organization. Preparation and use of food-based dietary guidelines. Geneva: WHO; 1998. Report of a Joint FAOMHO Consultation. [cited 2010 Mar 31]. Available from: <http://www. fao.org/docrep/X0243E/x0243e00.htm>.

21. Newby PK, Muller D, Hallfrisch D, Andres R, Tucker $\mathrm{KL}$. Food patterns measured by factor analysis and anthropometric changes in adults. Am J Clin Nutr. 2004; 80(2):504-13.

22. Tanner JM. Growth at adolescence. Oxford: Blackwell; 1962.

23. Must A, Dallal GE, Dietz WH. Reference data for obesity: $85^{\text {th }}$ and $95^{\text {th }}$ percentiles of body mass index (wt/ht2) and triceps skinfold thickness. Am J Clin Nutr. 1991; 53(4):839-46.

24. Willet WC, Howe GR, Kushi LH. Adjustments for total energy intake in epidemiologic studies. Am J Clin Nutr. 1997; 65(4 Suppl):1220S-8S.

25. O'Connor BP. SPSS and SAS programs for determining the number of components using parallel analysis and Velicer's MAP test. Behav Res Methods, Instr Comput. 2000; 32:396-402.

26. Li SJ, Paik HY, Joung H. Dietary patterns are associated with sexual maturation in Korean children. Br J Nutr. 2006; 95(4):817-23. doi: 10.1079/BJN20051675.

27. Silva SA, Santos PNS, Moura EC. Associação entre excesso de peso e consumo de feijão em adultos. Rev Nutr. 2010; 23 (2): 239-50. doi: 10.1590/ S1415-52732010000200007.

28. Shin KO, Oh SY, Park HS. Empirically derived major dietary patterns and their associations with overweight in Korean preschool children. Br J Nutr. 2007; 98(2):416-21.

29. Okubo H, Sasaki S, Murakami K, Kim MK, Takahashi $Y$, Hosoi $Y$, et al. Three major dietary patterns are all independently related to the risk of obesity among 3760 Japanese women aged 18-20 years. Int J Obes. 2008; 32(3):541-9. doi: 10.1038/sj.ijo. 0803737.

30. Kourlaba G, Panagiotakos DB, Mihas K, Alevizos A, Marayannis K, Mariolis A, et al. Dietary patterns in relation to socio-economic and lifestyle characteristics among Greek adolescents: a multivariate analysis. Public Health Nutr. 2009; 12(9):1366-72. doi: 10.1017/\$13689800080040 60.

31. Berg C, Lappas G, Wolk A, Strandhagen E, Torén $K$, Rosengren $A$, et al. Eating patterns and portion size associated with obesity in a Swedish population. Appetite. 2009; 52(1):21-6. doi: 10.10 16/j.appet.2008.07.008.

32. Summerbell CD, Moody RC, Shanks J, Stock MJ, Geissler C. Relationship between feeding pattern and body mass index in 220 free-living people in four age groups. Eur J Clin Nutr. 1996; 50(8):513-9.

33. Craig LCA, McNeill G, MacDiarmid JI, Masson LF, Holmes BA. Dietary patterns of school-age children in Scotland: association with socio-economic indicators, physical activity and obesity. $\mathrm{Br} J$ Nutr. 2010; 10(3):319-34.

34. Willett WC. Nutritional epidemiology. $2^{\text {nd }}$ ed. Oxford: Oxford University Press; 1998.

35. Majem LS, Bartrina JA. Introducción a la epidemiologia nutricional. In: Majem LS, Bartrina JA, Mataix-Verdú J. Nutrición y salud pública. Barcelona: Masson; 1995. p.59-65.

36. Freedman LS, Potischman N, Kipnis V, Midthune D, Schatzkin A, Thompson FE, et al. A comparison of two dietary instruments for evaluating the fat-breast cancer relationship. Int J Epidemiol. 2006; 35(4):1011-21. doi: 10.1093/ije/dyl085.

Recebido em: 3/8/2010

Versão final reapresentada em: 10/11/2010 Aprovado em: 31/5/2011 